\title{
Preliminary Screening for Microsatellite Instability and Loss of Heterozygosity in the Deleted In Colorectal Cancer (DCC) Gene among Filipino Patients with Colorectal Adenocarcinoma
}

\author{
Michael Ernesto S. Arnante, ${ }^{1,2}$ Ma. Luisa D. Enriquez, ${ }^{1,3}$ Ernelea P. Cao ${ }^{2}$ and Filipinas F. Natividad ${ }^{1}$ \\ ${ }^{1}$ Molecular Cytogenetics Laboratory, Research and Biotechnology, St. Luke's Medical Center, Quezon City, Philippines \\ ${ }^{2}$ Institute of Biology, University of the Philippines Diliman, Quezon City, Philippines \\ ${ }^{3}$ Department of Biology / Center for Natural Sciences and Ecological Research, De La Salle University, Manila, Philippines
}

\begin{abstract}
Objective. This study aimed to detect the presence of microsatellite instability (MSI) and loss of heterozygosity ( $\mathrm{LOH})$ of the Deleted in Colorectal Cancer (DCC) gene in normal and tumor tissues of Filipino colorectal cancer patients and examine its correlation with age, gender, tumor grade, tumor stage and site of lesion.
\end{abstract}

Methods. Paired frozen normal and tumor tissues from thirtynine (39) patients with colorectal adenocarcinoma were used in this study. Genomic DNA was extracted and amplified by polymerase chain reaction (PCR). Single strand conformation polymorphism - polyacrylamide gel electrophoresis (SSCP PAGE) was used to determine MSI and restriction fragment length polymorphism (RFLP) was used to study $\mathrm{LOH}$.

Results. Based on our data, out of the 39 patients, 10 showed $\mathrm{LOH}$ of the DCC gene using the $\mathrm{LOH}$ markers VNTR, M2 and M3, while no MSI was detected in the samples using the MSI markers BAT25 and BAT26. Correlation with clinicopathological characteristics showed that there is significance for the site of lesion. The $\mathrm{LOH}$ has correlation with tumor samples from the colon but not with those from the rectum.

Conclusion. Preliminary screening for MSI and $\mathrm{LOH}$ of the DCC gene shows that occurrence of colorectal cancer among Filipino patients can be correlated with $\mathrm{LOH}$ of the DCC gene but not with MSI. This may be the first study to examine the correlation or lack thereof of MSI and LOH of the DCC gene with colorectal cancer in a Filipino sample population.

Key Words: DCC gene, loss of heterozygosity, microsatellite instability, colorectal cancer

\footnotetext{
Presented as a poster at the 16th Asia-Pacific International Molecular Biology Network (A-IMBN) Annual Conference, December 3-5, 2013, Biopolis, Singapore.

Corresponding author: Michael Ernesto S. Arnante, MSc

Molecular Cytogenetics Laboratory

Research and Biotechnology Division

St. Luke's Medical Center

279 E. Rodriguez Sr. Blvd, Quezon City

1102 Philippines

Telephone: +6327275540

Email: mesarnante@gmail.com
}

\section{Introduction}

Colorectal cancer (CRC) development is marked by the growth of a benign precursor lesion from which a small percentage will progress into a carcinoma. Genetic alterations underlying the adenoma to carcinoma transition have been extensively studied over the years. ${ }^{1-3}$ In 1990, Fearon and Vogelstein proposed a genetic model for colorectal carcinogenesis in which they describe the event as a multi-step accumulation of mutational activation of oncogenes coupled with the inactivation of tumor suppressor genes driving the transformation from normal mucosa to adenoma to carcinoma. These genetic alterations eventually result in uninhibited cell growth, proliferation and tumor development. ${ }^{4}$ In the case of CRCs, two distinct genetic alterations are commonly observed; the first is microsatellite instability, which is detected in about $15 \%$ of all colorectal cancers, and the second involves chromosomal instability and loss of heterozygosity, which comprises almost $60-70 \%$ of the reported cases. ${ }^{5}$

Microsatellite instability (MSI) is defined as an alteration in the length of a microsatellite sequence within tumor DNA when compared with normal DNA from an individual. ${ }^{6}$ As a consequence of mispairings, insertions or deletions, MSI arises from changes in the number of units of mono-, di-, and trinucleotide DNA repeats which, due to their repetitive nature, are prone to errors during replication. Because this type of alteration can be detected only if many cells are affected by the same change in length, it is an indicator of the clonal expansion that is typical of a neoplasm. ${ }^{7}$ On the other hand, $\mathrm{LOH}$ refers to a change from a state of heterozygosity in a normal genome to a homozygous state in a paired tumor genome. ${ }^{8}$ It is usually described in the context of oncogenesis, where a mutational inactivation occurs in one of the allele of a tumor suppressor gene that is passed on to the zygote resulting in an offspring that is heterozygous for that allele, thus, the remaining functional allele in a somatic cell of the offspring becomes inactivated by mutation. It then results in nonproduction of tumor suppressor proteins leading to tumorigenesis. $\mathrm{LOH}$ has been shown to mark the site of tumor suppressor genes. ${ }^{9}$ 
Chromosome $18 \mathrm{q}$ is among the regions that harbor a tumor suppressor gene that is frequently inactivated by loss of heterozygosity during the development of several cancer types, including those of the gastrointestinal tract. In addition, colorectal cancers with $18 \mathrm{q}$ LOH have been shown to have a more aggressive clinical behavior than those without $18 \mathrm{q}$ LOH. ${ }^{10}$ The DCC gene was the first candidate tumor suppressor gene, identified in chromosome $18 \mathrm{q} .{ }^{11}$ While the DCC gene is expressed in most normal tissues, including colonic mucosa, its expression was greatly reduced or absent in most colorectal cancers tested. This was the first of several studies which implicated the DCC gene in the pathogenesis of colorectal cancer. At present, the DCC gene's exact mechanism of action in colorectal cancer is still unknown. Much of the reported data on loss and inactivation of the DCC gene is circumstantial. ${ }^{12}$ The traditional pathway suggested by Fearon and Vogelstein in 1990 states that the deletion $18 \mathrm{q}$ where the DCC gene is located mediates the transformation from early adenoma to late adenoma by interfering with apoptosis, allowing accumulation of mutations that hinders cell-cycle arrest or programmed cell death. Early studies have shown that the absence of DCC in tumors is linked to poor survival among patients with colorectal cancer. ${ }^{13}$ In contrast, later studies showed that molecular genetic changes do not appear to be useful prognostic markers in colorectal cancers. ${ }^{14}$

This study aims to provide additional information regarding the DCC gene's role in colorectal carcinogenesis, not only as a sole gene acting on cellular transformation from early adenoma to late adenoma but also as a part of a whole plethora of mutations occurring in tumorigenesis. It also aims to describe the molecular events that bring about the causes and progression of colorectal cancer in the context of Fearon and Vogelstein's model, with emphasis on the role of the DCC gene in colorectal adenocarcinoma formation. An updated assessment of the DCC gene in colorectal tumors is timely in order to further clarify the theories on its exact role in colorectal cancer development.

\section{Materials and Methods}

\section{Sample Collection}

Paired frozen normal and tumor tissues from 39 previously identified colorectal cancer patients who underwent surgical resection from 2000 to 2010 at St. Luke's Medical Center (SLMC), a tertiary hospital in the Philippines, were used in the study. Patients whose samples were used in this study have agreed to participate by previously signing consent forms before undergoing surgery. An information sheet and questionnaire were also given to them upon recruitment. All pertinent information such as age, gender and family history, among others, were entered in a databank. From this databank, all patient data including pathological information such as tumor stage, grade and surgical site were obtained for correlation and statistical analysis.

This protocol is under the umbrella project of SLMC entitled "A Molecular and Cytogenetic Study of Colorectal Cancer in the Philippine Population." This was reviewed by the Institutional Ethics Review Committee of SLMC (Reg. No. 07-033, Acc. No. 13-004).

\section{DNA Extraction}

All samples were previously stored in a $-80^{\circ} \mathrm{C}$ biofreezer. These were thawed at room temperature prior to processing. DNA extraction was carried out using QIAamp® DNA Mini Kit (QIAGEN, Germany) according to the manufacturer's instructions. Briefly, a lysis solution was used to disrupt the cell membrane to release DNA. Proteinase K was also added to the tissues to facilitate protein degradation. Tissue samples were incubated at $56^{\circ} \mathrm{C}$ for one to three hours or until tissue was completely lysed. Ethanol was added to precipitate the isolated DNA. Wash buffers were added separately to remove residual contaminants on the isolated genomic DNA. Purified genomic DNA was then eluted from the column. DNA quality and quantity were assessed using UV spectrophotometry. A $50 \mathrm{ng} / \mu \mathrm{L}$ of DNA served as working solution for PCR.

\section{Microsatellite Instability}

Analysis for MSI was performed using a modified procedure by Loukola et al. ${ }^{15}$ Two sets of primers from the Bethesda panel of markers were used, BAT25 and BAT26. They were chosen because they are the best-known mononucleotide markers for MSI testing. ${ }^{16}$ These markers can identify MSI even when the tumor content of a sample is as low as $5-10 \%$. PCR reactions were carried out in a $25 \mu \mathrm{l}$ reaction volume containing $50 \mathrm{ng}$ of genomic DNA, 1x PCR buffer, $250 \mu \mathrm{M}$ each deoxynucleotide triphosphate (dNTPs), $0.5 \mu \mathrm{M}$ each primer, and 1 unit of Taq polymerase. Optimized $\mathrm{MgCl}_{2}$ concentrations for BAT25 and for BAT26 were $2.5 \mathrm{mM}$ and $2.75 \mathrm{mM}$, respectively. PCR was performed under the following conditions:

- For BAT25, initial denaturation at $95^{\circ} \mathrm{C}$ for $10 \mathrm{~min}$, and 28 cycles of $95^{\circ} \mathrm{C}$ for $1 \mathrm{~min}, 56^{\circ} \mathrm{C}$ for $45 \mathrm{sec}$ and $72^{\circ} \mathrm{C}$ for $45 \mathrm{sec}$ and a final extension at $72^{\circ} \mathrm{C}$ for 10 $\min$.

- For BAT26, the PCR condition involved initial denaturation at $95^{\circ} \mathrm{C}$ for $10 \mathrm{~min}$ and 32 cycles of $95^{\circ} \mathrm{C}$ for $45 \mathrm{sec}, 55^{\circ} \mathrm{C}$ for $1 \mathrm{~min}$ and $72^{\circ} \mathrm{C}$ for $30 \mathrm{sec}$ and a final extension at $72^{\circ} \mathrm{C}$ for $10 \mathrm{~min}$. Single Strand Conformation PolymorphismPolyacrylamide Gel Electrophoresis (SSCP-PAGE) was performed to observe target bands.

Gels were photographed under white light after staining. Microsatellite instability was demonstrated by variation of band sizes of PCR products of tumor samples in comparison with normal samples. 
Loss of Heterozygosity $(\mathrm{LOH})$

Analysis for $\mathrm{LOH}$ was performed using the modified procedure by Gao et al ${ }^{17}$ and Akkiprik et al. ${ }^{18}$ PCR reactions were carried out in a $25 \mu \mathrm{l}$ reaction volume containing $50 \mathrm{ng}$ of genomic DNA, 1x PCR buffer, $250 \mu \mathrm{M}$ each of dNTPs, 2.5 $\mathrm{mM} \mathrm{MgCl}$, $0.5 \mu \mathrm{M}$ each primer, and 1 unit of Taq polymerase. To check for cross contamination, a sample without template DNA and only having distilled water served as the negative control. PCR reactions were run using the following conditions: $5 \mathrm{~min}$ of initial denaturation at $95^{\circ} \mathrm{C}$, 35 cycles of $95^{\circ} \mathrm{C}$ for $1 \mathrm{~min}$ denaturation, $54^{\circ} \mathrm{C}$ for 45 sec for annealing and $72^{\circ} \mathrm{C}$ for $30 \mathrm{sec}$ extension, and 5 minutes of final extension at $72^{\circ} \mathrm{C}$.

Three markers were used to determine $\mathrm{LOH}$. These were the variable number tandem repeat (VNTR) region of chromosome 18, and the M2 and M3 sites of intron 5 of DCC. For the VNTR region, PCR products were separated directly on 2.5\% agarose gels. For the M2 and M3 polymorphisms, $7 \mu \mathrm{l}$ of PCR products were mixed with $10 \mathrm{U}$ of the restriction enzyme MspI and incubated at $37^{\circ} \mathrm{C}$ overnight. These were separated on $2 \%$ agarose gels.

\section{Statistical Analysis}

Statistical analysis using SPSS (ver.17.0) was performed for all categorical variables namely: gender (male vs. female), age (>50 vs. $<50)$, site of lesion (colon vs. rectum), histological grade $(1,2,3$ or 4$)$, stage $(1,2,3$ or 4$)$, MSI (microsatellitestable or MSS, microsatellite-low or MSI-L, microsatellitehigh or MSI-H) and DCC LOH (negative vs. positive). Fisher's exact tests were used for calculation. Results were considered statistically significant when the two-sided $p$ value is less than 0.05 .

\section{Results}

Clinicopathological data acquired from the Colorectal Cancer Databank at SLMC are summarized in Table 1. Two patients with no clinicopathological data were excluded in the statistical analysis.

Table 1. Summary of clinicopathological characteristics and presence of MSI and LOH.

\begin{tabular}{|c|c|c|c|c|c|c|c|c|c|c|c|}
\hline & \multirow[t]{2}{*}{ Field \# } & \multirow[t]{2}{*}{ Sex } & \multirow[t]{2}{*}{ Age } & \multirow[t]{2}{*}{ Grade } & \multirow[t]{2}{*}{ Stage } & \multirow[t]{2}{*}{ Site of Lesion } & \multicolumn{2}{|c|}{ MSI } & \multicolumn{3}{|c|}{ LOH } \\
\hline & & & & & & & BAT25 & BAT26 & VNTR & M2 & M3 \\
\hline 1 & 320 & $\mathrm{M}$ & 63 & 2 & 3 & colon & no & no & & het & $\mathrm{LOH}$ \\
\hline 2 & 342 & $\mathrm{~F}$ & 55 & 2 & 4 & rectum & no & no & & hom & hom \\
\hline 3 & 220 & M & 80 & 2 & 2 & colon & no & no & no & het & hom \\
\hline 4 & 260 & M & 69 & 2 & 2 & colon & no & no & no & hom & hom \\
\hline 5 & 436 & M & 47 & 2 & 2 & rectum & no & no & no & het & hom \\
\hline 6 & 402 & $\mathrm{~F}$ & 73 & 2 & 2 & colon & no & no & no & hom & hom \\
\hline 7 & 268 & M & 78 & 2 & 2 & colon & no & no & no & hom & hom \\
\hline 8 & 355 & M & 59 & 2 & 3 & rectum & no & no & no & het & hom \\
\hline 9 & 369 & M & 54 & 2 & 2 & colon & no & no & no & hom & $\mathrm{LOH}$ \\
\hline 10 & 413 & & & & & & no & no & no & het & $\mathrm{LOH}$ \\
\hline 11 & 426 & M & 52 & 2 & 3 & rectum & no & no & no & hom & hom \\
\hline 12 & 326 & M & 67 & 2 & 2 & colon & no & no & no & hom & $\mathrm{LOH}$ \\
\hline 13 & 365 & $\mathrm{~F}$ & 62 & 2 & 3 & colon & no & no & no & hom & hom \\
\hline 14 & 421 & $\mathrm{M}$ & 58 & 2 & 3 & colon & no & no & no & & hom \\
\hline 15 & 329 & M & 59 & 2 & 2 & rectum & no & no & no & & hom \\
\hline 16 & 349 & $\mathrm{~F}$ & 72 & 2 & 3 & colon & no & no & no & & hom \\
\hline 17 & 428 & $\mathrm{~F}$ & 29 & 4 & 3 & colon & no & no & $\mathrm{LOH}$ & hom & $\mathrm{LOH}$ \\
\hline 18 & 309 & $\mathrm{~F}$ & 37 & 1 & 3 & rectum & no & no & no & hom & hom \\
\hline 19 & 417 & & & & & & no & no & no & het & hom \\
\hline 20 & 449 & $\mathrm{~F}$ & 54 & 2 & 3 & rectum & no & no & no & hom & hom \\
\hline 21 & 454 & $\mathrm{~F}$ & 52 & 2 & 1 & colon & no & no & no & hom & hom \\
\hline 22 & 444 & M & 47 & 4 & 3 & colon & no & no & no & het & $\mathrm{LOH}$ \\
\hline 23 & 368 & $\mathrm{~F}$ & 40 & 2 & 2 & colon & no & no & $\mathrm{LOH}$ & het & hom \\
\hline 24 & 203 & $\mathrm{~F}$ & 72 & 1 & 3 & colon & no & no & no & het & het \\
\hline 25 & 507 & M & 76 & 2 & 3 & rectum & no & no & no & het & hom \\
\hline 26 & 508 & M & 52 & 4 & 3 & colon & no & no & no & het & hom \\
\hline 27 & 517 & M & 59 & 4 & 2 & colon & no & no & $\mathrm{LOH}$ & hom & hom \\
\hline 28 & 569 & $\mathrm{M}$ & 82 & 2 & & colon & no & no & no & $\mathrm{LOH}$ & hom \\
\hline 29 & 523 & $\mathrm{M}$ & 74 & 4 & 1 & colon & no & no & no & hom & hom \\
\hline 30 & 522 & M & 63 & 4 & 2 & colon & no & no & no & hom & hom \\
\hline 31 & 519 & $\mathrm{~F}$ & 58 & 2 & 4 & colon & no & no & no & het & hom \\
\hline 32 & 518 & $\mathrm{~F}$ & 72 & 4 & 4 & rectum & no & no & no & hom & hom \\
\hline 33 & 509 & M & 48 & & & rectum & no & no & no & het & hom \\
\hline 34 & 566 & $\mathrm{M}$ & 24 & 1 & & rectum & no & no & no & het & hom \\
\hline 35 & 560 & M & 54 & & 3 & rectum & no & no & no & het & hom \\
\hline 36 & 529 & $\mathrm{~F}$ & 53 & 2 & 3 & colon & no & no & no & het & het \\
\hline 37 & 525 & M & 30 & & & colon & no & no & no & het & hom \\
\hline 38 & 570 & M & 58 & 2 & & colon & no & no & $\mathrm{LOH}$ & het & hom \\
\hline 39 & 574 & $\mathrm{M}$ & 83 & & & & no & no & no & hom & hom \\
\hline
\end{tabular}

Legend: LOH - presence of loss of heterozygosity; het - heterozygous for the corresponding marker; hom - homozygous for the corresponding marker 


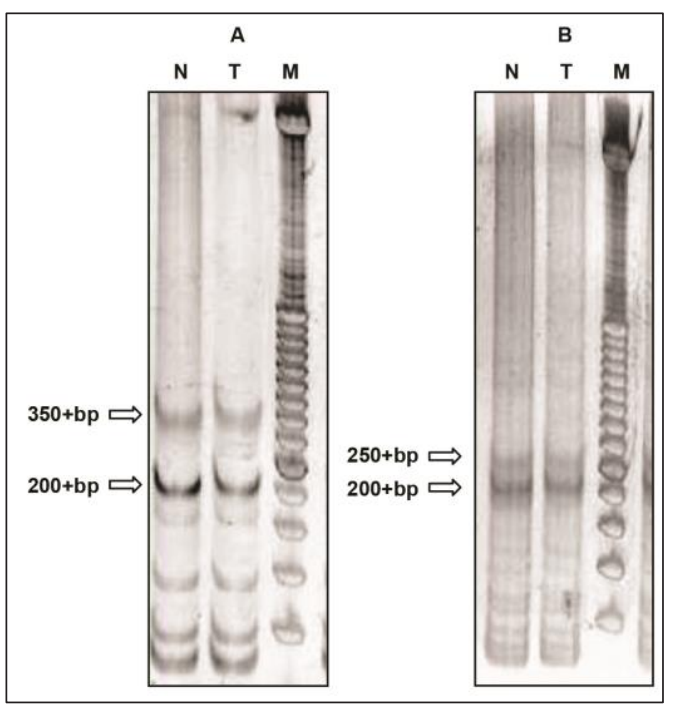

Figure 1. Representative gels for MSI. A: BAT25 B: BAT26. Legend: N: normal tissue, T: tumor tissue, M: molecular marker ladder.

For MSI testing, change in the band size between the normal and tumor samples indicates positive microsatellite instability. None of the paired samples in our study showed microsatellite instability for the two markers. For BAT25, the standard two target bands (200 bp and $350 \mathrm{bp}$ ) were observed in both normal and tumor samples (Figure 1a). For BAT26, the standard two target bands (200 bp and $250 \mathrm{bp}$ ) were also observed in both normal and tumor samples (Figure 2b).

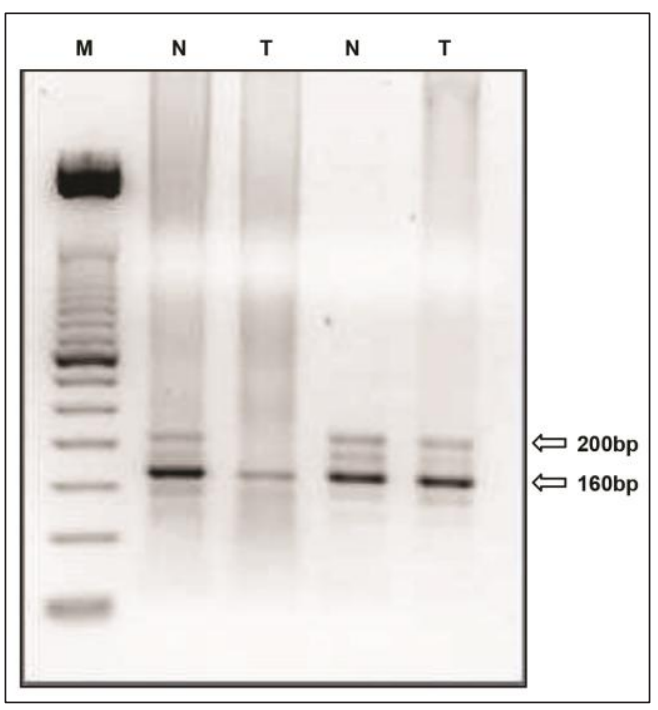

Figure 2. Representative gel for VNTR.

Legend: M: molecular marker ladder, N: normal tissue, T: tumor tissue

For LOH, three known sites were observed for analysis. These were the VNTR, M2 and M3 sites. For the VNTR sites, several bands were observed ranging from sizes $160-200 \mathrm{bp}$. Each patient had a different number of bands for the VNTR site. Loss of one or more of these bands in the tumor sample indicated presence of LOH (Lanes 2-3, Figure 2). Absence of $\mathrm{LOH}$ is shown when the normal and tumor sample had no change in resulting bands (Lanes 4-5, Figure 2).

For the M2 site, a homozygous sample revealed either a single band at $396 \mathrm{bp}$ (Figure 3, M2-A) or two bands at 257

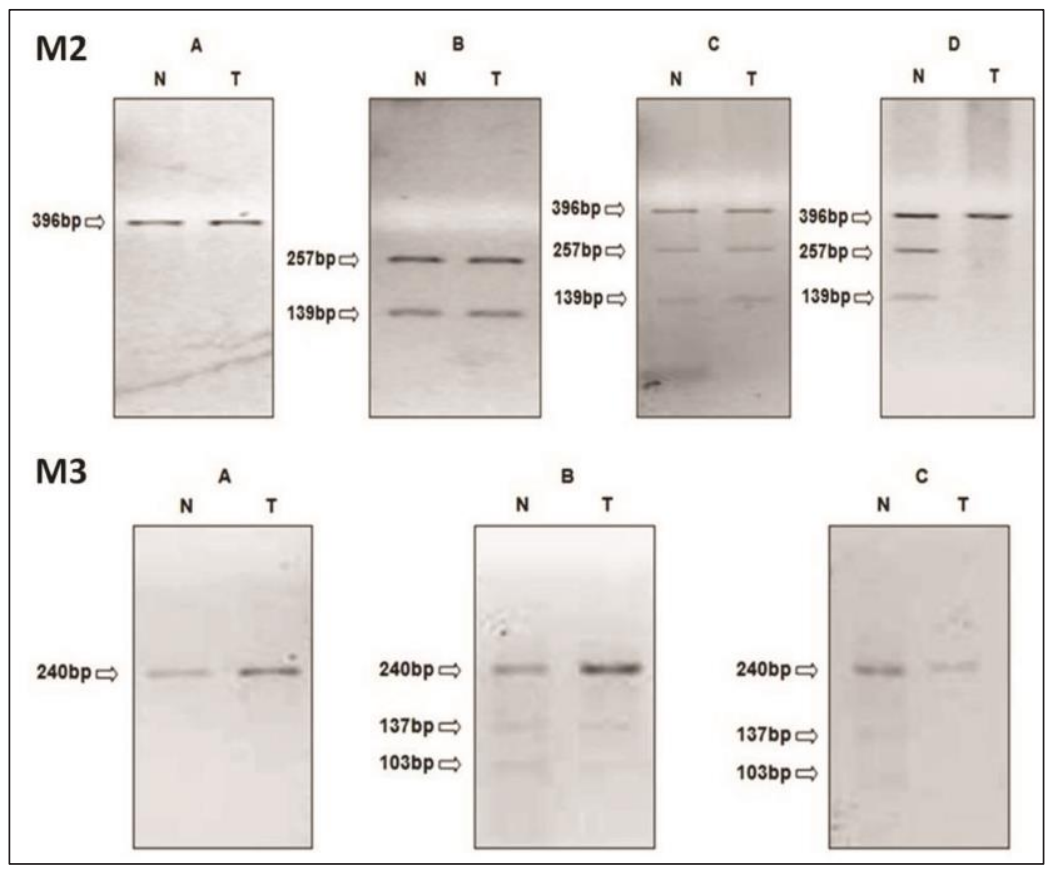

Figure 3. Representative gels for M2 AND M3 polymorphism. Legend: N: normal tissue, T: tumor tissue 
bp and 139 bp (Figure 3, M2-B). A heterozygous sample showed presence of all three bands (Figure 3, M2-C). Loss of the two bands at $257 \mathrm{bp}$ and $139 \mathrm{bp}$ was seen in the tumor sample while the normal counterpart showed heterozygosity (Figure 3, M2-D).

For the M3 site, a homozygous sample revealed a single band at $240 \mathrm{bp}$ (Figure 3, M3-A). A heterozygous sample showed presence of three bands at $240 \mathrm{bp}, 137 \mathrm{bp}$ and $103 \mathrm{bp}$ (Figure 3, M3-B). Loss of the two bands at $137 \mathrm{bp}$ and $103 \mathrm{bp}$ was seen in the tumor sample while the normal counterpart showed heterozygosity (Figure 3, M3-C).

A positive $\mathrm{LOH}$ of the $\mathrm{DCC}$ gene was assessed by $\mathrm{LOH}$ at one or any combination of these three sites. A total of 10 samples were found positive for $\mathrm{LOH}$. Table 1 shows three samples with $\mathrm{LOH}$ for only the VNTR marker, one sample showed LOH for only the M2 marker, and five samples showed LOH for the M3 marker. One sample showed a combination of LOH in the M3 and VNTR markers. Only nine samples with $\mathrm{LOH}$ were used for correlation since one sample had an incomplete clinicopathological data.

Statistical analysis of results was done using Fisher's exact test in SPSS. Results were considered statistically significant when $\mathrm{p}$ value $<0.05$. Samples were grouped according to age $(\leq 50$ or $>50)$, gender (male or female), tumor grade (1, 2, 3 or 4$)$, tumor stage (1, 2, 3 or 4 ) and site of lesion (colon or rectum). They were then analyzed for correlation with the presence or absence of LOH in the DCC gene (Table 2). Analysis showed that the presence or absence of $\mathrm{LOH}$ had significant correlation at the site of lesion in the colon area $(p=0.018)$. No LOH was seen in samples where the lesion was in the rectal area.

Table 2. Statistical analysis. Comparison of clinicopathological characteristics and LOH.

\begin{tabular}{|c|c|c|c|c|c|c|c|c|}
\hline \multirow{2}{*}{ Variables } & \multicolumn{2}{|c|}{ With LOH } & \multicolumn{2}{|c|}{ Without LOH } & \multirow{2}{*}{ Total } & \multicolumn{3}{|c|}{ Fisher's exact test } \\
\hline & Count & Row \% & Count & Row \% & & $\mathrm{x}^{2}$ & $\mathrm{df}$ & P value \\
\hline Age & & & & & & --- & --- & 0.655 \\
\hline$<=50$ & 3 & 33.3 & 6 & 66.7 & 9 & & & \\
\hline$>50$ & 6 & 20.7 & 23 & 79.3 & 29 & & & \\
\hline Total & 9 & 23.7 & 29 & 76.3 & 38 & & & \\
\hline Sex & & & & & & --- & --- & 0.438 \\
\hline Female & 2 & 14.3 & 12 & 85.7 & 14 & & & \\
\hline Male & 7 & 29.2 & 17 & 70.8 & 24 & & & \\
\hline Total & 9 & 23.7 & 29 & 76.3 & 38 & & & \\
\hline Grade & & & & & & 1.998 & 2 & $0.368^{*}$ \\
\hline 1 & 0 & 0.0 & 3 & 100.0 & 3 & & & \\
\hline 2 & 6 & 26.1 & 17 & 73.9 & 23 & & & \\
\hline 4 & 3 & 42.9 & 4 & 57.1 & 7 & & & \\
\hline Total & 9 & 27.3 & 24 & 72.7 & 33 & & & \\
\hline Stage & & & & & & 2.711 & 3 & $0.438^{*}$ \\
\hline 1 & 0 & 0.0 & 2 & 100.0 & 2 & & & \\
\hline 2 & 4 & 36.4 & 7 & 63.6 & 11 & & & \\
\hline 3 & 3 & 20.0 & 12 & 80.0 & 15 & & & \\
\hline 4 & 0 & 0.0 & 3 & 100.0 & 3 & & & \\
\hline Total & 7 & 22.6 & 24 & 77.4 & 31 & & & \\
\hline Site of Lesi & ion & & & & & --- & --- & $0.018^{*}$ \\
\hline Colon & 9 & 36.0 & 16 & 64.0 & 25 & & & \\
\hline Rectum & 0 & 0.0 & 12 & 100.0 & 12 & & & \\
\hline Total & 9 & 24.3 & 28 & 75.7 & 37 & & & \\
\hline
\end{tabular}

\section{Discussion}

The importance of the DCC gene has been a longstanding issue in the molecular basis of the pathway of colorectal carcinogenesis. The studies by Fearon et al demonstrated that while the DCC gene was expressed in most normal tissues, including colonic mucosa, its expression was greatly reduced or absent in most colorectal carcinomas. ${ }^{4}$ In addition, allelic losses of $18 \mathrm{q}$ are infrequent in early stage tumors but are common in primary colorectal carcinomas and nearly $100 \%$ of hepatic metastases arising from colorectal primaries, implying that chromosome $18 \mathrm{q}$ loss may contribute more to progression rather than initiation of colorectal cancer. These early discoveries opened up the possibility that the DCC gene may play a role in the pathogenesis of human colorectal neoplasia, with an initial hypothesis that it alters the normal cell-cell interactions controlling growth and proliferation of colorectal cancer cells.

In the case of MSI, Saletti et al reported that only 15\% of sporadic colorectal cancers show presence of MSI. ${ }^{6}$ In addition, Samowitz et al reported that MSI in sporadic colon cancer is associated with an improved prognosis at the population level. ${ }^{19}$ They performed MSI testing using BAT26 as non-coding mononucleotide repeat and the transforming growth factor- $\beta$ receptor type II as the coding mononucleotide repeat. Using a genetic analyzer, they found significant relationships between MSI and clinicopathological factors such as proximal tumor location, gender, age at diagnosis, poor histological differentiation, and low tumor stage. In our study, we did not detect any MSI in all our samples. It is possible that minute differences in base pairing may be present but could not be detected by the method used in this study (SSCP-PAGE) as compared to the genetic analyzer which provided more sensitive resolution in terms of difference in band sizes.

As for the LOH, several studies have reported that there is significant correlation in terms of clinicopathological characteristics of patients. In the work of Chetty et al they observed that allelic imbalance of the DCC gene occurs more frequently in patients less than 50 years of age as compared to those who are 50 years and above. ${ }^{20}$ However, their study did not find any correlation with other clinicopathological parameters.

Our study has shown significant relationship only for the site of lesion but not with other clinicopathological characteristics. Previous studies have not reported any association between $\mathrm{LOH}$ of the DCC gene and at the site of lesion like in the work done by Matsuzaki et al ${ }^{21}$ but just to point out, they separated the group between those that had lesions in the right and left colon instead of between the colon and rectum. Other studies have used other techniques like immunohistochemistry on paraffin-embedded tissue sections to study association of the DCC gene expression with clinicopathological parameters, ${ }^{22}$ and in this case, they 
observed a difference in the gene expression in the site of lesion between colon and rectum. Our results were almost similar with what they have reported.

The results of this study provide new insights on the DCC gene, especially in the Filipino population. Unpublished studies involving mutation profile analysis of the $K$-ras and p53 genes showed that high frequency of mutations occur outside the known hotspots, suggesting a different profile for Filipino patients (Nuylan and Enriquez, 2008; Pilante and Enriquez, 2010). Since there are no previously reported associations between $\mathrm{LOH}$ and site of lesion, this is the first report of such an association in the Filipino population.

A review article of Cortez and Leonardo ${ }^{23}$ emphasized the need to recognize high-risk groups, primary preventive measures and promotion of screening tests. They stressed the importance of genetic profiling to identify high-risk groups to be able to institute a valid and rational program of surveillance.

Due to the small sample size, the correlations seen in this study need to be validated in a similar study with a larger number of samples. As most previous researchers have reported association of the DCC gene with characterization of tumor stage, the absence of correlation in our study has to be confirmed with a larger sample size.

Though no significant association with the tumor stage was observed, the results of this study still provide useful insights on the management of colorectal carcinogenesis especially among Filipino population. For instance, the molecular composition of colorectal tumors may be different, depending on the site of lesion whether in the colon or the rectum. This makes an impact on the decision of the physician on how to treat and manage his patient.

\section{Conclusion}

The observation that there is a correlation between $\mathrm{LOH}$ of the DCC gene and site of lesion contributes to establishing the molecular mechanisms that underlie the pathways for colorectal carcinogenesis. Understanding the characteristics of colorectal cancer at the molecular level is critical in formulating possible methods of diagnosis and treatment for patients. Differential treatment for patients where the lesion occurs in the colon using targeted therapies may thus be possible.

\section{References}

1. Tsang $\mathrm{AH}$, Cheng $\mathrm{KH}$, Wong AS, et al. Current and future molecular diagnostics in colorectal cancer and colorectal adenoma. World J Gastroenterol. 2014; 20(14):3847-57.

2. Brooks D, Winawer S, Rex D, et al. Colonoscopy surveillance after polypectomy and colorectal cancer resection. Am Fam Physician. 2008; 77(7): 924-6.

3. Hanahan D, Weinberg R. The hallmarks of cancer. Cell. 2000; 100(1):5770 .
4. Fearon E, Cho K, Nigro J, et al. Identification of a chromosome 18q gene that is altered in colorectal cancers. Science. 1990; 247(4398):49-56.

5. Boland CR, Goel A. Microsatellite instability in colorectal cancer. Gastroenterology. 2010; 138(6):2073-87.

6. Saletti P, Edwin I, Pack K, et al. Microsatellite instability: application in hereditary non-polyposis colorectal cancer. Ann Oncol. 2001; 12(2):15160.

7. de la Chapelle A. Microsatellite Instability. New Engl J Med. 2003; 349:209-10.

8. Beroukhim R, Lin M, Park Y, et al. Inferring loss-of-heterozygosity from unpaired tumors using high-density oligonucleotide SNP arrays. PLoS Comput Biol. 2006; 2(5):e41.

9. Asimakopolous FA, Gilbert JG, Aldred MA, Pearson TC, Green AR. Interstitial deletion constitutes the major mechanism for loss of heterozygosity on chromosome 20q in polycythemia vera. Blood. 1996; 88(7):2690-8.

10. Fearon E, Pierceall W. The Deleted in Colorectal Cancer (DCC) Gene: A candidate tumour suppressor gene encoding a cell surface protein with similarity to neural cell adhesion molecules. Cancer Surv. 1995; 24:3-17.

11. Mehlen P,Fearon E. Role of the dependence receptor DCC in colorectal cancer pathogenesis. J Clin Oncol. 2004; 22(16): 3420-8.

12. Worthley DL, Whitehall VL, Spring KJ, Leggett BA. Colorectal carcinogenesis: Road maps to cancer. World J Gastroenterol. 2007; 13(28):3784-91.

13. Shibata D, Reale M, Lavin P, et al. The DCC protein and prognosis in colorectal cancer. N Engl J Med. 1996; 335(23):1727-32.

14. Zauber N, Wang C, Lee P, et al. Ki-ras gene mutations, LOH of the APC and DCC genes, and Microsatellite Instability in primary colorectal carcinoma are not associated with micrometastases in pericolonic lymph nodes or with patients' survival. J Clin Pathol. 2004; 57:938-42.

15. Loukola A, Eklin K, Laiho P, et al. Microsatellite marker analysis in ccreening for Hereditary Nonpolyposis Colorectal Cancer (HNPCC). Cancer Res. 2001; 61:4545-9.

16. Brennetot C, Buhard O, Jourdan F, et al. Mononucleotide repeats BAT26 and BAT-25 accurately detect MSI-H tumors and predict tumor content: Implications for population screening. Int J Cancer. 2005; 113:446-50.

17. Gao X, Honn KV, Grignon D, Sakr W, Chen YQ. Frequent loss of expression and loss of heterozygosity of the putative tumor suppressor gene DCC in prostatic carcinomas. Cancer Res. 1993; 53:2723-7.

18. Akkiprik M, Ataizi-Celikel C, Dusunceli F, et al. Clinical significance of p53, K-ras and DCC gene alterations in the stage I-II colorectal cancers. J Gastrointestin Liver Dis. 2007; 16(1):11-7.

19. Samowitz W, Curtin K, Ma K, et al. Microsatellite instability in sporadic colon cancer is associated with an improved prognosis at the population level. Cancer Epidimiol Biomarkers Prev. 2001; 10:917-23.

20. Chetty R, Naidoo R and Schneider J. Allelic imbalance and microsatellite instability of the DCC gene in colorectal cancer in patients under the age of 35 using fluorescent DNA technology. Mol Pathol. 1998; 51:35-8.

21. Matsuzaki K, Deng G, Tanaka H, et al. The relationship between global methylation level, loss of heterozygosity and microsatellite instability in sporadic colorectal cancer. Clin Cancer Res. 2005; 11(24):8564-9.

22. Sun X, Rutten S, Zhang H, et al. Expression of the deleted in colorectal cancer gene is related to prognosis in DNA diploid and low proliferative colorectal adenocarcinoma. J Clin Oncol. 1999; 17(6):1745-50.

23. Cortez E, Leonardo M. Comprehensive review of published articles on colon and rectal cancer in the Philippines, 1980-2002. St. Luke's J Med. $2004 ; 2: 3-8$. 